\title{
Shapes of obstacles in the longitudinal road profile
}

\author{
Oldřich Kropáča and Peter Múčka ${ }^{\mathrm{b}, *}$ \\ a Scientific Consultant, Kaňkovského 1241/2, CZ-182 00 Prague 8, Czech Republic \\ ${ }^{\mathrm{b}}$ Research worker, Institute of Materials and Machine Mechanics, Slovak Academy of Sciences, Račianska 75, \\ SK-831 02 Bratislava 3, Slovak Republic
}

Received 22 December 2009

Revised 11 May 2010

\begin{abstract}
A voluminous set of longitudinal road profiles gathered from the Long Term Pavement Performance (LTPP) program was processed using median filtering to separate individual large obstacles from the basic quasi-homogeneous random road unevenness. The shapes of 16590 obstacles were analysed partitioned into four sub-groups according to the asphalt-concrete vs. cement-concrete road surfaces, and positive (bumps) vs. negative (potholes) vertical elevations. The empirical obstacle shapes were fitted using seven symmetric and two asymmetric analytical shapes. Based on the root mean square error (RMSE) criterion and accounting for some practical aspects connected with the possible use for the simulation of road profiles with obstacles generalized parabolic shapes (power function) and half-sine shapes seem to be recommendable as adequate approximation functions. Asymmetric obstacle shapes of cosine and parabolic shapes were also briefly discussed and their impact on the vibration transient response of a quarter-car model outlined.
\end{abstract}

Keywords: Road profile, vibration response, obstacle shape, bump, pothole, quarter-car model, long term pavement performance program

\section{Introduction}

One of the most frequently appearing vibration/shock acting on people in their everyday activity stems from passing a motor vehicle over a road/terrain profile imperfections. The knowledge of real shapes of road obstacles is necessary for solving various problems in a road-vehicle-operator interaction system. Surprisingly, no comprehensive study identifying the empirical shapes of road obstacles were published yet. This question is important for many engineering fields concerned for example with vehicle dynamics, vibration measurement and testing, shock testing and isolation, structural dynamics, biodynamics, whole-body vibration, etc.

An optimally working road transport system necessitates a high cooperation of two basic sub-components of the transport system, i.e. high road performance ability and considerate dynamical properties of travelling vehicles. As for the roads concerns, their performance ability is predominantly assessed according to the quality of the pavement surface. Among several indicators, which specify very different features of the road surface, the longitudinal road unevenness (roughness) have a major impact. Two main components of road unevenness may be distinguished: continuous (and usually homogeneous along the wheelpath) unevenness of random nature, and individual large obstacles (OB) or other local distresses appearing mainly randomly along the wheelpath. In spite of rather rare occurrence of the latter, their detrimental effect is crucial because they cause devastating impulse loadings to the pavement structure.

\footnotetext{
*Corresponding author. Tel.: +421 25930 9402; Fax: +421 25477 2099; E-mail: ummsmuc@savba.sk.
} 
The authors elaborated different partial features of the obstacle problem in papers [6-9] where references to other authors dealing with this topic are summarized and therefore are not repeated here. Only additional references dealing with the obstacle shapes are cited. The papers [6-9] do not solved the problem of the obstacles' shapes. In the papers [6,7], the analytical obstacle shapes (trapezoidal and cosine) and obstacles' dimensions without any connection with the obstacles of the real road profiles were used. The obstacle dimensions were chosen approximately with considering of following relation between obstacle length $\left(l_{O B}\right)$ and height $\left(h_{O B}\right), l_{O B}=50 h_{O B}$ while the heights were considered from the interval $0.5-4 \mathrm{~cm}$. The aim was to estimate the influence of obstacles superimposed on the random road profile on commonly used road unevenness statistical descriptors [6] and the vehicle vibration response [7]. The papers [8,9] describe the separation of obstacles from the continuous random unevenness component using median filtering. Exploiting the LTPP program database [5], statistics and probability density functions (PDFs) of six geometric indicators of obstacles (length, height, ratio length to height, area, volume and distance between obstacles) were evaluated.

The shapes of obstacles are tackled only sporadically in the literature. Pesterev et al. [13] consider a half-sine function approximated by a parabola and a cosine function approximated by a 4th degree polynomial. Öijer and Edlund [12] selected a pothole elliptic shape from the analysis of front axle acceleration response of a finite element tractor-trailer model. In Mitschke et al. [11], half-sine is used for individual large obstacles, a sequence of sinuses for corrugation, saw-form for periodical subsidence of joins and many of structural obstacles due to necessary technical solutions on the pavement surface (transverse drainage channel, manhole cover, bridge transition). Ferris [3] has processed approximately $10^{6}$ obstacle events on road surfaces ranging from highways to gravel roads to derive partial components, into which a large global longitudinal profile encompassing obstacles may be decomposed. Czech technical standard CSN 300560 [1] uses two different bumps of circular segment shape and pothole of trapezoidal shape for testing of vehicle vibration dynamic response.

Nevertheless, no correlations are available between such analytical models and populations of empirical shapes appearing on in-service roads. Thus, the aim of this study is the following:

(a) Comparison of a set of analytical shapes with the mean of in situ measured obstacle shapes;

(b) Identification of differences in obstacle shapes in dependence on the vertical orientation of local obstacles (bump/pothole) and material used for the pavement surface (asphalt/cement concrete);

(c) Estimation of possible asymmetry in obstacle shapes;

(d) Comparison of vertical vibration response of a standard vehicle model caused by various analytical shapes of obstacles.

The recommendations for the simulation of road surfaces with prescribed obstacles will be outlined.

\section{Separation of obstacles from the longitudinal road profile by median filtering}

Median is a position indicator of a random variable alternative to the generally applied mean value. Given a series $\left\{x_{j}\right\}$, this sequence is upwardly ordered, i.e., it holds $x_{(j)} \leqslant x_{(k)}$ for $j<k, j=0,1,2, \ldots, 2 N$. Then, $x_{(k)}$ is the $k$-th order statistics of the series $\left\{x_{j}\right\}$. The mean value of the ordered sequence $\left\{x_{(j)}\right\}$, i.e., $x_{(N)}$ is the estimate of its median at the $j$-th position of the series $\left\{x_{j}\right\}$. When shifting the window along the original random sequence and conduction ordering of data in each step, a median filter as the simplest case from a more general family of statistical filters is obtained. It follows from the definition of the median filter that for the complete filtering of the non-random component embracing $m$ discrete data, the $n \geqslant 2 m+1$ window length is needed. Then, the median filter works as a low-pass filter in a similar way as a linear filter.

Ordering of data is a strong nonlinear operation, but variant programs providing estimation of median are part of commercial statistical program packages. In the Matlab ${ }^{\circledR}$ environment, the program medfitt 1 with the single parameter $n$ as the filter window length plays this role.

Figure 1 shows the principle of generation of the median-filtered sequence $h_{M F}\left(l_{j}\right)$ from the primarily given sequence $h\left(l_{j}\right)$. Removing of both bump and pothole obstacles are indicated.

Median filters have some other important properties that differentiate them from the linear filters (for details see e.g., [4]). For our application task, it is important that they are capable to remove large obstacles from the longitudinal road profile without affecting the neighbourhood of the random unevenness component. 


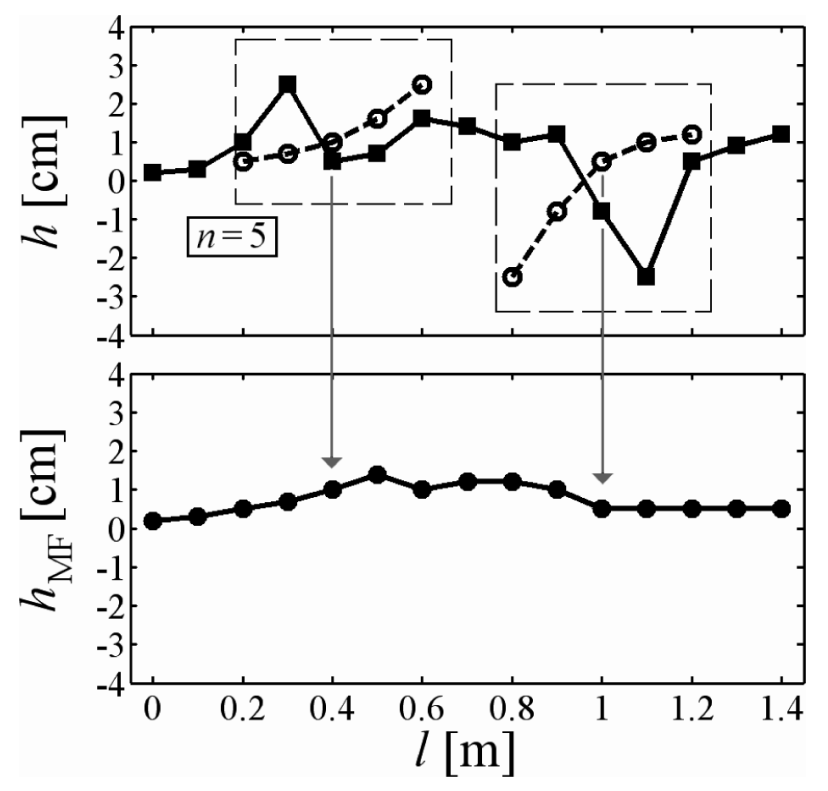

Fig. 1. Principle of a median filter.

From the measured road profile $h\left(l_{j}\right)$, exploiting the medfilt 1 program, the obstacle profile $h_{O B}\left(l_{j}\right)$ was obtained in the form

$$
h_{O B}\left(l_{j}\right)=h\left(l_{j}\right)-h_{M F}\left(l_{j}\right),
$$

where $h_{M F}\left(l_{j}\right)=\operatorname{medfilt} 1\left(h\left(l_{j}\right), n\right)$ is an estimate of the cleaned-up random part (i.e. median-filtered sequence using the $n$-th order median filter) obtained by the medfilt 1 program.

To obtain better distinguishing ability of obstacle parameters, the original sections sampling with $\Delta l=0.1524 \mathrm{~m}$ step was re-sampled with $1 \mathrm{~cm}$ while applying linear interpolation. At the same time, some further formal provisions were conducted to enable unambiguous estimation of geometric obstacle parameters (for details see [8]).

\section{Road data used}

For the analysis of unevenness, data from the LTPP program were used [5]. Only sections from general pavement studies (GPS) were considered. The LTPP program started in 1987 with the aim at collecting data on roads, highways and motorways from all US states and some Canadian provinces over a 20 year periods. Test sections from this research program are 500 feet $(L=152.4 \mathrm{~m})$ long. Their profiles were measured using K.J. Law profilometers and recorded with the distance step 0.5 feet (i.e., $\Delta l=0.1524 \mathrm{~m}$ ) thus giving 1000 measured points per section. The road type (highway, motorway, minor road) indication for particular test section identification numbers was not provided so the correlation to road type was not possible. Therefore the roads were separated only into two groups according to their wearing courses, namely asphalt cement (AC), and Portland concrete cement (CC). All the measured profiles with their detailed description are available in $[2,5]$.

It must be noted that these LTPP data resulted from the pre-processing of primary data collected at $2.54 \mathrm{~cm}$ distance intervals with a $30.48 \mathrm{~cm}$ long moving average (MA) filter applied before downsampling to $0.1524 \mathrm{~m}$ distance intervals. Due to this pre-processing, especially that of the MA filter, a substantial distortion of the basic obstacle dimensions appeared the quantification of which is presented in [8]. The possible impact on the obstacle shape will be discussed in Section 5.1.

In summary, 23402 profile records were analyzed ( $\mathrm{AC}-14104, \mathrm{CC}-9298$ ). Obstacles were detected in 5036 records (AC - 3628, CC - 1408) from which parameters of 16590 obstacles (13563 potholes, 3027 bumps) were evaluated and classified as follows: $\mathrm{AC}+\mathrm{P}-11857, \mathrm{AC}+\mathrm{B}-1884, \mathrm{CC}+\mathrm{P}-1706, \mathrm{CC}+\mathrm{B}-1143$. The obstacles 
Table 1

Analytical functions $h_{N}=f\left(l_{N}\right)$ for fitting the normalised obstacles (upper signs refer to bumps, lower ones to potholes)

\begin{tabular}{ll}
\hline Obstacle shape & Analytical function \\
\hline cos & $\pm 0.5\left(1-\cos \left(2 \pi l_{N}\right)\right)$ \\
half-sine & $\pm \sin \left(\pi l_{N}\right)$ \\
half-ellipse & $\pm \sqrt{1-4\left(l_{N}-0.5\right)^{2}}$ \\
power(1) & $\mp\left(2\left|1_{N}-0.5\right|-1\right)$ \\
power(1.25) & $\mp\left(2.3784\left|1_{N}-0.5\right|^{1.25}-1\right)$ \\
power(1.5) & $\mp\left(2.8284\left|1_{N}-0.5\right|^{1.5}-1\right)$ \\
power(1.75) & $\mp\left(3.3636\left|1_{N}-0.5\right|^{1.75}-1\right)$ \\
power(2) & $\mp\left(4\left(1_{N}-0.5\right)^{2}-1\right)$ \\
\hline
\end{tabular}

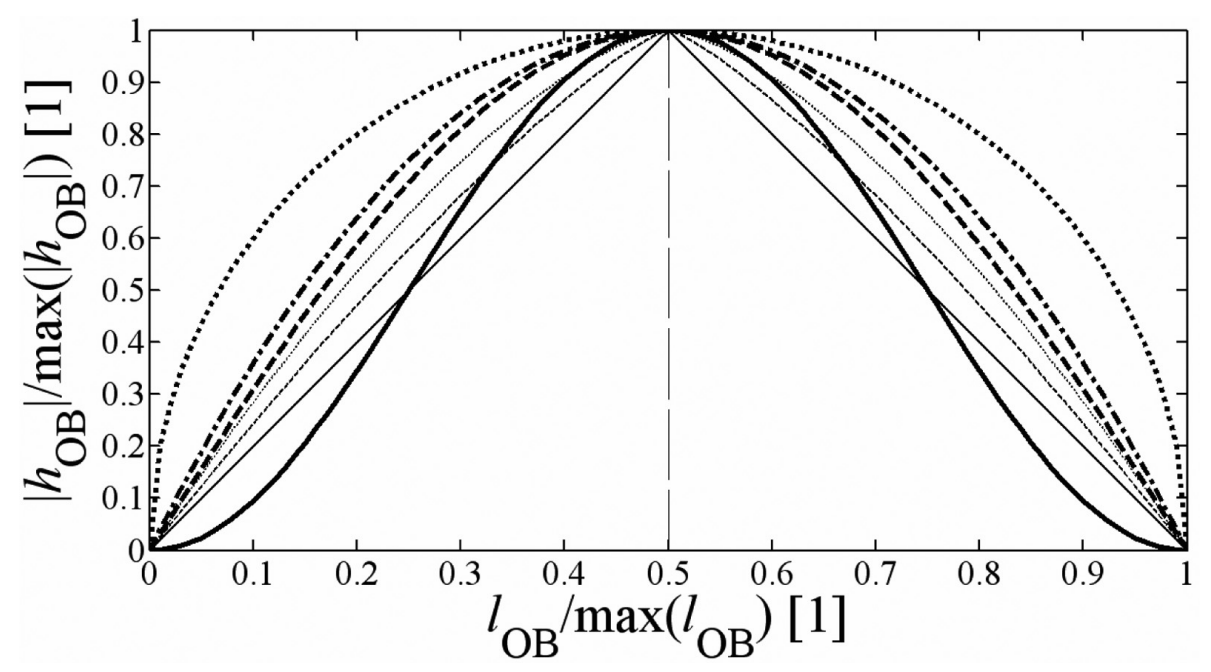

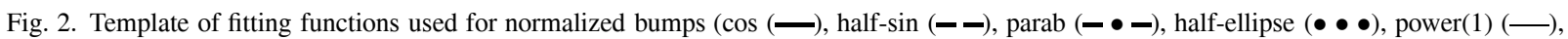

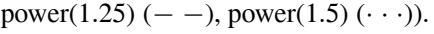

took on many different forms. Thus for a simplified denomination: bump (B) is used for each positive, and pothole (P) for each negative obstacle. As obstacles, local differences in terrain profile greater than absolute threshold value $3 \mathrm{~mm}$ were taken into account. Further, the window length for median filter was considered of order, $n=13(n \Delta l=$ $1.98 \mathrm{~m})$, which approximately corresponds to the double of the estimated maximum obstacle length $(\sim 1 \mathrm{~m})$ in the processed road sections. Some kinds of distress, such as narrow transverse cracking, or joint spalling, could not be captured. Statistics and probability density functions of all obstacle parameters (height, length, etc.) are given in paper [8].

\section{Selection of analytical models for obstacle shapes}

Based on data given in the literature and own practical experience, in Table 1, analytical functions suitable for fitting obstacle shapes are summarized. Besides the most frequently used functions cosine, half-sine and half-ellipse, a power function (generalized parabola) family with variable power is considered, which further encompasses two simple analytical functions, triangle (with power one, i.e. $k=1$ ) and parabola (with power two, i.e. $k=2$ ). All the presented functions are normalized for unity length and unity maximum height $\left(h_{N}=h_{O B} / \max \left(h_{O B}\right)\right.$, $\left.l_{N}=l_{O B} / \max \left(l_{O B}\right)\right)$, while upper sign relates to bumps, and lower sign to potholes. Plotting all these functions into a common rectangular, a template (Fig. 2) is obtained to which all the empirical shapes may be compared, and closeness between analytical and empirical shapes in terms of root mean square error (RMSE) and correlation coefficients (R) established. RMSE is commonly used statistics for evaluating the overall quality of a regression 
Table 2

Fitting parameters for various analytical models of obstacles

\begin{tabular}{|c|c|c|c|c|c|c|c|c|}
\hline \multirow[t]{2}{*}{ Obstacle analytical shape } & \multicolumn{2}{|c|}{$\mathrm{AC}+\mathrm{B}(1884)$} & \multicolumn{2}{|c|}{$\mathrm{CC}+\mathrm{B}(1143)$} & \multicolumn{2}{|c|}{$\mathrm{AC}+\mathrm{P}(11857)$} & \multicolumn{2}{|c|}{$\mathrm{CC}+\mathrm{P}(1706)$} \\
\hline & RMSE & $\mathrm{R}$ & RMSE & $\mathrm{R}$ & RMSE & $\mathrm{R}$ & RMSE & $\mathrm{R}$ \\
\hline $\cos$ & 0.2163 & 0.863 & 0.2350 & 0.802 & 0.1706 & 0.909 & 0.2379 & 0.780 \\
\hline half-sine & 0.1452 & 0.889 & 0.1789 & 0.832 & 0.1410 & 0.911 & 0.1933 & 0.810 \\
\hline half-ellipse & 0.2194 & 0.853 & 0.2466 & 0.805 & 0.2535 & 0.851 & 0.2645 & 0.784 \\
\hline power(1) & 0.1900 & 0.868 & 0.2053 & 0.807 & 0.1482 & 0.909 & 0.2067 & 0.784 \\
\hline power(1.25) & 0.1581 & 0.880 & 0.1820 & 0.820 & 0.1259 & 0.915 & 0.1889 & 0.798 \\
\hline power(1.5) & 0.1437 & 0.887 & 0.1740 & 0.828 & 0.1262 & 0.915 & 0.1853 & 0.806 \\
\hline power(1.75) & 0.1423 & 0.889 & 0.1758 & 0.832 & 0.1391 & 0.911 & 0.1902 & 0.810 \\
\hline parabola & 0.1488 & 0.888 & 0.1833 & 0.833 & 0.1569 & 0.904 & 0.1995 & 0.811 \\
\hline Range & 0.0771 & 0.036 & 0.0726 & 0.031 & 0.1276 & 0.064 & 0.0792 & 0.031 \\
\hline
\end{tabular}

model. RMSE refers to the differences between values predicted by a model and the measured data and is calculated as square root of residual sum of squares, divided by the number of degrees of freedom. Note: To conform to the real height/length ratio of the obstacle, the height-unity is plotted in the absolute dimension as a half of the length-unity.

\section{Processing of obstacle shapes}

Processing of obstacles extracted from the LTPP database specified in detail in [8] revealed that there are noticeable differences in basic obstacle dimensions between bumps (B) vs. potholes (P) and asphalt concrete (AC) vs. Portland cement concrete $(\mathrm{CC})$ road surfaces. Therefore, for the processing of the population of profiles, four partial subpopulations were selected, viz. $\mathrm{AC}+\mathrm{B}, \mathrm{AC}+\mathrm{P}, \mathrm{CC}+\mathrm{B}$, and $\mathrm{CC}+\mathrm{P}$. The results of testing the closeness of all partial sub-populations are presented in Table 2, root mean square error, RMSE, and correlation coefficient, R, are used for the quantification of differences between empirical obstacle shapes and their fitting functions. A visual comparison is displayed in Fig. 3 and only a reduced number of most important analytical shapes are drawn to hold the picture lucid.

The fitting of empirical obstacles data was conducted using support functions for nonlinear regression implemented in Matlab (nlinfit, nlparci, nlintool). Nonlinear least-squares data fitting by the Gauss-Newton method was used. All the computations were made under Matlab ${ }^{\circledR}$ support.

\subsection{Effect of the moving average pre-processing of LTPP data on obstacle shape distortion}

As noticed earlier, LTPP data used for the obstacle specification were pre-processed using a MA filter what resulted in a distortion of the basic obstacle dimensions. Due to normalizing of the template in Fig. 2 to the $1 \times 1$ format, dimension distortions are irrelevant. Nevertheless, the above-mentioned MA pre-processing, too, could affect the obstacle shapes.

Therefore, a study has been conducted in which two most used obstacle shapes were considered, viz. the parabola (i.e. power(2)) and cosine. In Fig. 4(a), besides the primary and filtered shapes also the power $(k)$ with $k=1.75,1.5$ and 1.25 are plotted for comparison. It appears that the MA-filtered profile lies across power(1.25) and power(1.75) with a mean shape power(1.5) making the shape somewhat wide. Inversely, a constant 0.5 should be added to the $k$ parameter obtained from LTPP data. Nevertheless, reflecting the very near closeness of RMSE and R parameters of different obstacle shapes as presented in Table 2, such a difference may be neglected. In Fig. 4(b), similar comparison is given for a cosine shape. In contrast to the preceding case, the MA-filtered shape is somewhat widely. As in the case of power $(k)$, this change of the obstacle shape may be neglected reflecting the great variability of the processed empirical LTPP shapes used for comparison.

\section{Asymmetric obstacle shapes}

According to Table 2, only negligible differences between suitable fitting functions seem, but in Fig. 3, asymmetric shapes on CC pavements are apparent. Therefore, the actual position $a$ on the $l_{N}$ axis belonging to the peak values 


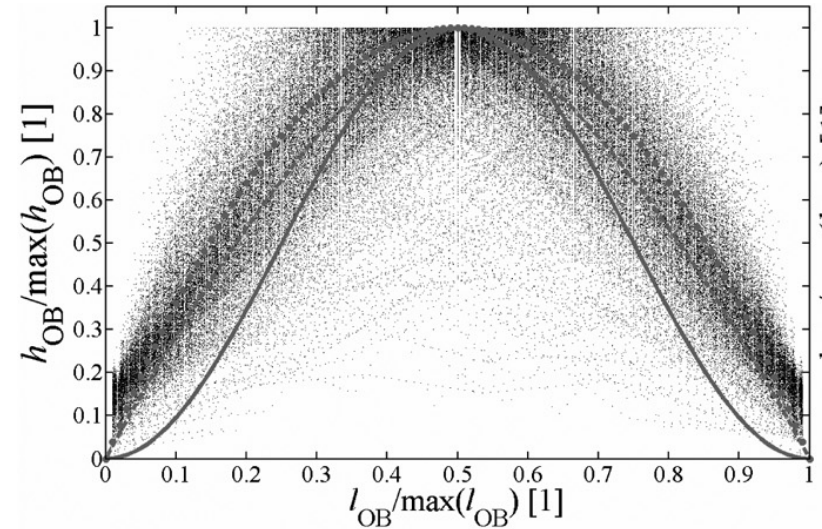

(a)

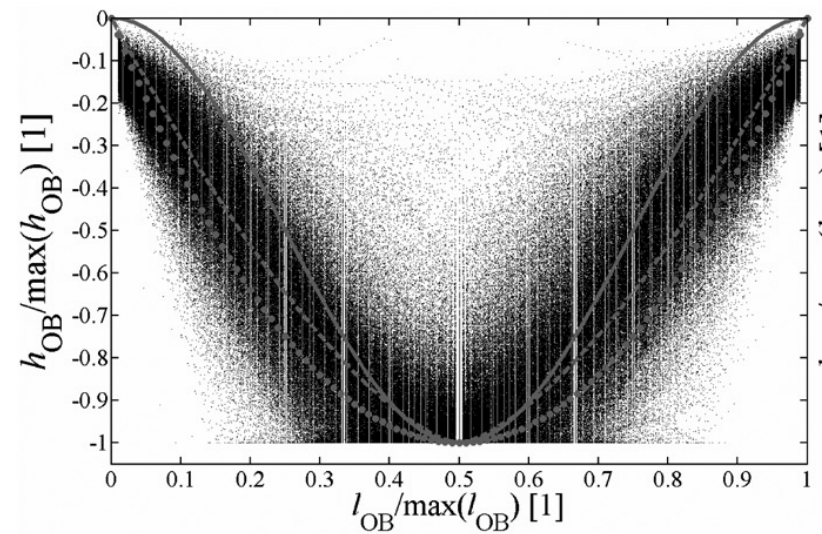

(c)

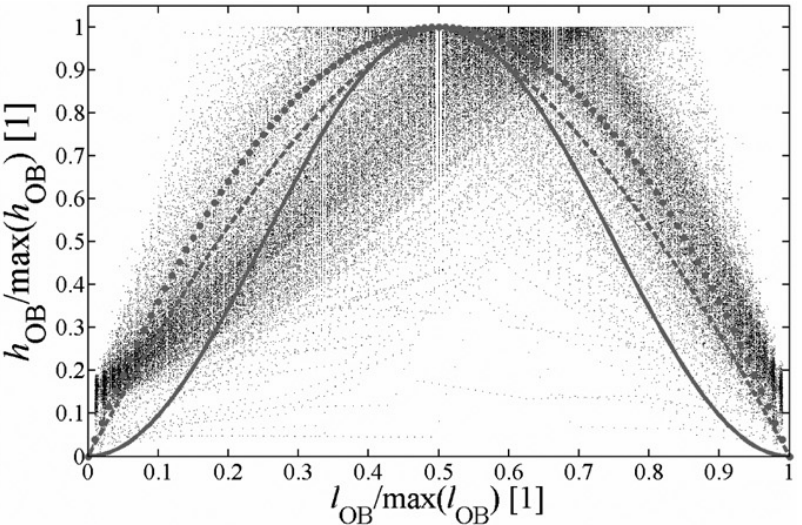

(b)

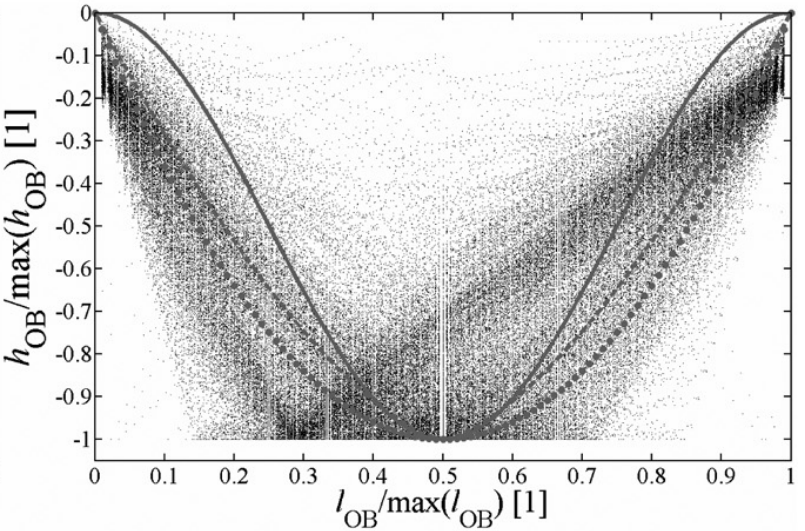

(d)

Fig. 3. Visual comparison of normalized empirical obstacles $(\cdots)$ with some analytical fitting functions (cos $(-)$, power(1.5) $(--)$, parabola ( . )): (a) $\mathrm{AC}+\mathrm{B}$; (b) $\mathrm{CC}+\mathrm{B}$; (c) $\mathrm{AC}+\mathrm{P}$; (d) $\mathrm{CC}+\mathrm{P}$.

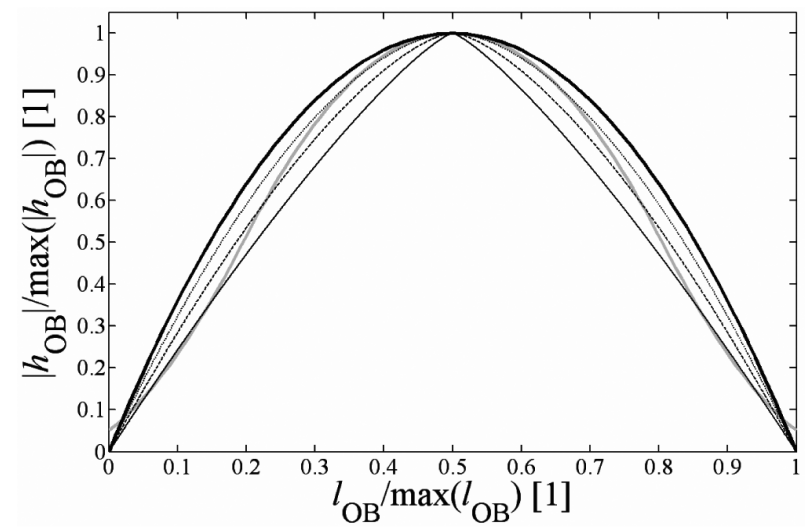

(a)

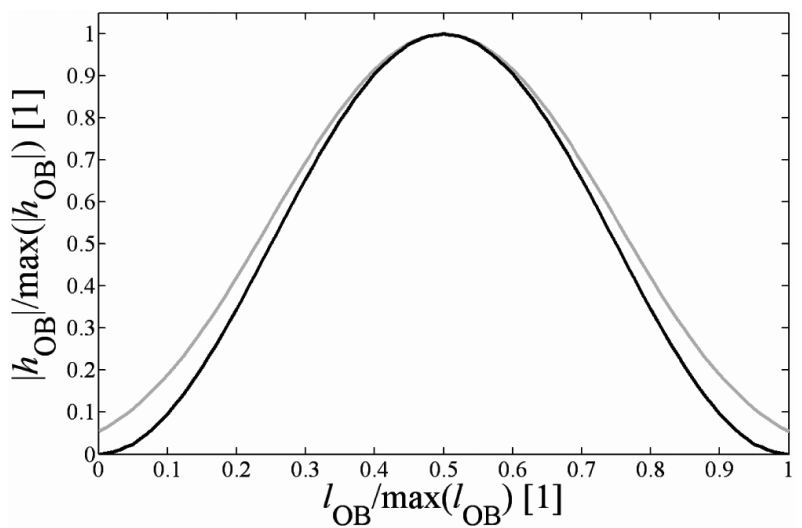

(b)

Fig. 4. Comparison of primary and MA-filtered obstacle shapes: (a) parabolic (-), MA-filtered parabolic (-), power(1.25) (-), power(1.5) $(--)$, power $(1.75)(\cdots)$; (b) cosine $(-)$, MA-filtered cosine $(-)$. 
Table 3

Statistics of extreme values positions $a$ on the $l_{N}$ axis

\begin{tabular}{lcccc}
\hline & MEAN & MEDIAN & MODUS & $\operatorname{std}(a)$ \\
\hline AC+Bumps (1884) & 0.496 & 0.494 & 0.490 & 0.129 \\
CC+Bumps (1143) & 0.585 & 0.613 & 0.710 & 0.138 \\
AC+Potholes (11857) & 0.483 & 0.483 & 0.490 & 0.097 \\
CC+Potholes (1706) & 0.425 & 0.417 & 0.290 & 0.143 \\
\hline
\end{tabular}

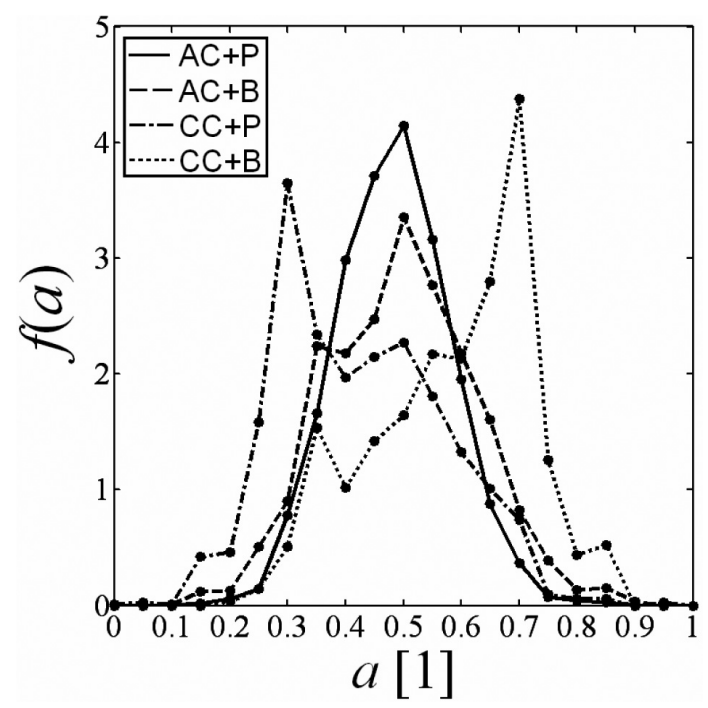

Fig. 5. PDFs of the positions $a$ of extreme values of obstacles.

of empirical shapes is demonstrated with mean (MEAN), median (MED), modal (MOD), and STD values in Table 3 and on probability density functions (PDF) in Fig. 5.

For AC road surface, symmetric models for obstacle shapes proved to be acceptable both for bumps and potholes. On the contrary, for CC road surface distinct asymmetric shapes seem to be apparent, in addition with opposite skew values for bumps and potholes. Therefore, a separate examination of asymmetric obstacle shapes was conducted as follows.

\subsection{Asymmetric generalized power function}

To obtain suitable fitting functions for these generalized shapes, the power $(k)$ function was selected as most versatile. Nevertheless, it did not succeed to find a close enough fitting function with one $k$ parameter common for the whole obstacle length. Therefore, the normalised length of the obstacle was divided onto two parts, encompassing the onset (OS) and run-out (RO) branches. The approximation functions for both branches were formulated separately in the form

$$
\begin{array}{llll}
O N-S E T: & l_{\mathrm{N}} \in\langle 0, a\rangle & h_{\mathrm{N}}=\mp\left(b_{O S}\left|l_{\mathrm{N}}-a\right|^{k_{O S}}-1\right), & b_{O S}=1 / a^{k_{O S}} \\
R U N-O U T: & l_{\mathrm{N}} \in(a, 1\rangle & h_{\mathrm{N}}=\mp\left(b_{R O}\left|l_{\mathrm{N}}-a\right|^{k_{R O}}-1\right), & b_{R O}=1 /(1-a)^{k_{R O}}
\end{array}
$$

For the location of obstacle peak values $a$ common for both these branches their estimates for MEAN, MED and MOD as stated in Table 3 and Fig. 5 were used as fixed values. The reason is to avoid different estimates of $a$ when including them into two different regression relationships for OS and RO branches. Further, unknown $k$ and $b$ parameters for both branches were estimated separately by standard nonlinear regression in case of $k$ and according to above-given relationships for $b$ with an assumption, $\operatorname{RSTD}(b)=\operatorname{RSTD}(k)$. RSTD means the relative standard deviation, i.e. the absolute value of the ratio of the standard deviation to the mean value. The results are presented in Table 4 and Fig. 6. In Table 4, estimates of $k \mathrm{~s}$ with their $\operatorname{STDs}(k)$ express the uncertainties of $k \mathrm{~s}$ while the RMSE 
Table 4

Fitting parameters of asymmetric obstacle shapes power $\left(k_{O S}, k_{R O}\right)$ to real CC pavements separately for onset and run-out branches using different estimates of $a$

\begin{tabular}{llccccccc}
\hline Estimate of $a$ & & $a$ & $k$ & $\operatorname{std}(k)$ & $b$ & std $(b)$ & RMSE & $R$ \\
\hline MEAN & Bump (OS) & 0.585 & 1.3660 & 0.0034 & 2.0800 & 0.0038 & 0.1507 & 0.8690 \\
& Bump (RO) & 0.585 & 1.7612 & 0.0050 & 4.7065 & 0.0205 & 0.1389 & 0.8940 \\
& Pothole (OS) & 0.425 & 1.6254 & 0.0043 & 4.0181 & 0.0148 & 0.1653 & 0.8480 \\
\multirow{4}{*}{ MEDIAN } & Pothole (RO) & 0.425 & 1.3076 & 0.0029 & 2.0619 & 0.0034 & 0.1675 & 0.8370 \\
& Bump (OS) & 0.613 & 1.4589 & 0.0035 & 2.0421 & 0.0035 & 0.1483 & 0.8750 \\
& Bump (RO) & 0.613 & 1.6027 & 0.0046 & 4.5791 & 0.0202 & 0.1405 & 0.8910 \\
& Pothole (OS) & 0.417 & 1.5862 & 0.0042 & 4.0044 & 0.0147 & 0.1654 & 0.8480 \\
MODUS & Pothole (RO) & 0.417 & 1.3321 & 0.0029 & 2.0519 & 0.0033 & 0.1671 & 0.8380 \\
& Bump (OS) & 0.710 & 1.7811 & 0.0040 & 1.8404 & 0.0026 & 0.1466 & 0.8780 \\
& Bump (RO) & 0.710 & 1.0591 & 0.0037 & 3.7100 & 0.0168 & 0.1494 & 0.8670 \\
& Pothole (OS) & 0.290 & 0.9613 & 0.0031 & 3.2870 & 0.0124 & 0.1711 & 0.8180 \\
& Pothole (RO) & 0.290 & 1.7208 & 0.0036 & 1.8028 & 0.0022 & 0.1680 & 0.8400 \\
\hline
\end{tabular}

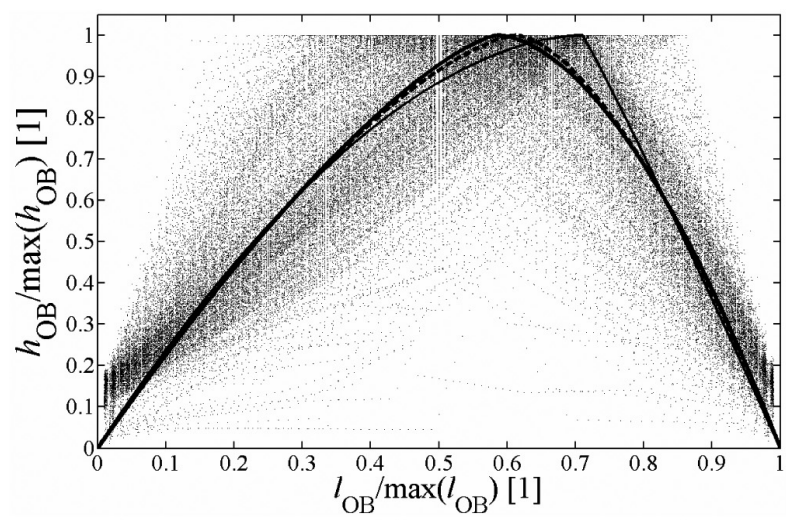

(a)

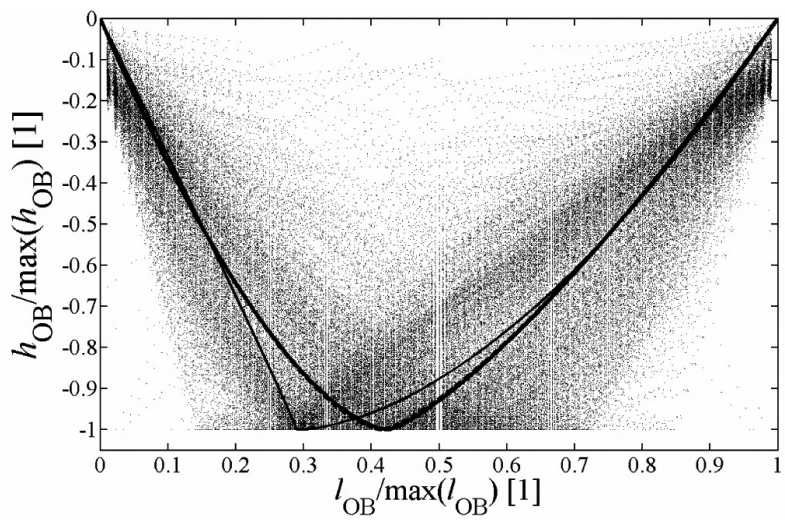

(b)

Fig. 6. Fitting of asymmetric obstacle shapes power( $a$ ) separately for OS/RO branches: (a) CC+B; (b) CC+P, using estimates of $a$ (mean ( $\longrightarrow$ ), median (- $)$, modus $(-))$.

and $\mathrm{R}$ in the last columns quantify the overall closeness between the set of empirical shapes and its particular model just specified.

The different results obtained for different $a$-estimates may be assessed as follows: The common statement is that distinct differences between bump and pothole obstacle shapes are apparent. The asymmetry, which follows from the MEAN $a$-estimate, is rather small but the $k$-values are rather different for OS and RO branches. Though this feature is not unrealistic, it is in some way inconsistent with the general demand on as simple-as-possible of analytical models. In this case one would expect that the $k_{O S}$ and $k_{R O}$ values would differ only within their uncertainty intervals. The MOD $a$-estimate takes rather high value and the differences between $k \mathrm{~s}$ for OS and RO branches are even higher than in the case of MEAN $a$-estimate. Moreover, both branches of the obstacle shape intersect at quite different slopes thus forming a sharp peak in the intersection point what is at distinct variance with the shapes of individual empirical obstacles. Therefore, this variant of obstacle parameters estimation should be judged as not acceptable. The remaining way of obstacle parameters estimation based on MED $a$-estimate provides a plausible $a$-value and small differences in $k$ estimates for both shape branches. This model could be recommended for possible simulation studies encompassing road profiles with large obstacles with asymmetric shapes as it has been detected for $\mathrm{CC}$ pavements.

\subsection{Asymmetric cosine shapes}

An asymmetric counterpart to the cosine shape, the following variant to the last-named has been derived in the form 
Table 5

Fitting closeness of asymmetric cosine obstacle shapes to real CC pavements

\begin{tabular}{lllll}
\hline Estimate of $a$ & & $a$ & RMSE & $R$ \\
\hline MEAN & Bump (OS) & 0.585 & 0.2013 & 0.857 \\
& Bump (RO) & 0.585 & 0.2225 & 0.864 \\
& Pothole (OS) & 0.425 & 0.2313 & 0.819 \\
MEDIAN & Pothole (RO) & 0.425 & 0.2126 & 0.823 \\
& Bump (OS) & 0.613 & 0.2056 & 0.860 \\
& Bump (RO) & 0.613 & 0.2095 & 0.870 \\
& Pothole (OS) & 0.417 & 0.2320 & 0.819 \\
MODUS & Pothole (RO) & 0.417 & 0.2124 & 0.825 \\
& Bump (OS) & 0.710 & 0.2308 & 0.848 \\
& Bump (RO) & 0.710 & 0.1889 & 0.860 \\
& Pothole (OS) & 0.290 & 0.2093 & 0.814 \\
& Pothole (RO) & 0.290 & 0.2424 & 0.803 \\
\hline
\end{tabular}

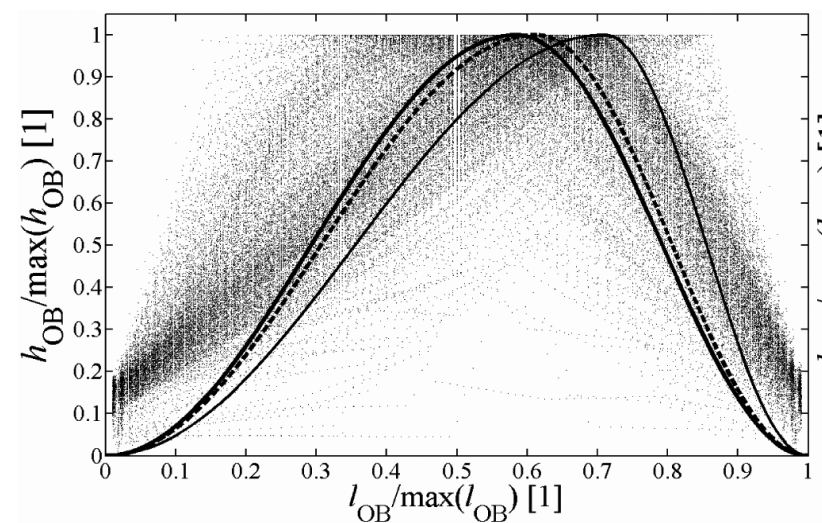

(a)

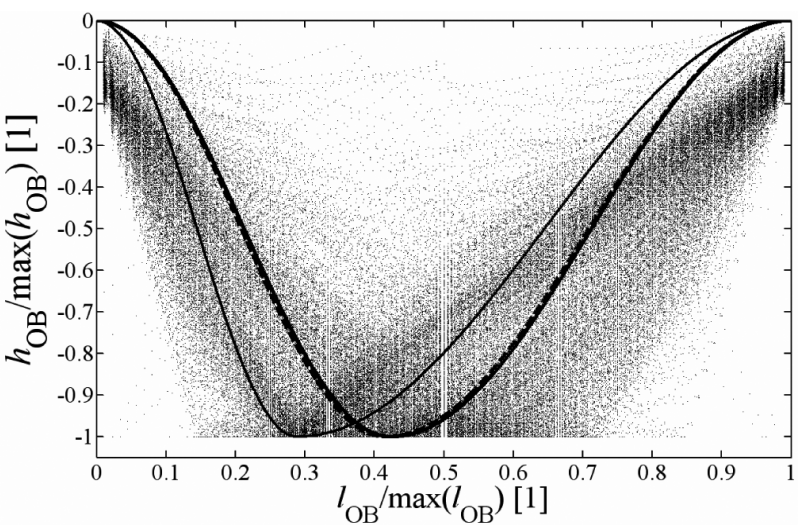

(b)

Fig. 7. Fitting of asymmetric COS obstacle shapes separately for OS/RO branches: (a) $\mathrm{CC}+\mathrm{B}$; (b) $\mathrm{CC}+\mathrm{P}$, using estimates of $a$ (mean ( - ), median (- $)$, modus (-)).

$$
\begin{array}{lll}
O N-S E T: & l_{\mathrm{N}} \in\langle 0, a) & h_{\mathrm{N}}= \pm 0.5\left(1-\cos \left(\frac{0.5}{a} 2 \pi l_{\mathrm{N}}\right)\right) \\
R U N-O U T: & l_{\mathrm{N}} \in(a, 1\rangle & h_{\mathrm{N}}= \pm 0.5\left(1+\cos \left(\frac{0.5}{1-a} 2 \pi\left(l_{\mathrm{N}}-a\right)\right)\right)
\end{array}
$$

where for the parameter $a$, the estimates obtained from the empirical CC data were used. The closeness of fitting is presented in Table 5 and the visual presentation in Fig. 7. Examining Fig. 7(b) it appears that a shape composed of three subsections would be closeness, viz. with COS for the OS-subsection defined for $a$ from 0 to $a_{1}=0.29$, constant value -1 for the subsection from $a_{1}$ to $a_{2}=0.425$ and a COS shape for the RO-subsection from $a_{2}$ to 1.0 . Such flat shapes with possibly alternatively defined $a_{1}, a_{2}$ values could be suitable e.g. for the ravelling type distress description.

\section{Effect of obstacle shapes on the dynamic response of quarter-car model}

The destroying effect of large obstacles manifests itself the response of over-passing vehicle when producing additional dynamic loading of its critical components. At the same time dynamic loading to the pavement also takes place. Therefore, a simulation study was conducted in which the vibration response of a quarter-car model with 2-degrees-of-freedom was pursued. The vehicle model parameters were applied in accordance with definition of the reference quarter-car model intended for calculation of the commonly used road unevenness indicator, International Roughness Index (IRI) [14] as follows: $m_{1}=15 \mathrm{~kg}, m_{2}=100 \mathrm{~kg}, k_{1}=65300 \mathrm{~N} / \mathrm{m}, k_{2}=6330 \mathrm{~N} / \mathrm{m}, b_{2}=$ 
Table 6

Statistics of quarter-car model vibration response to different obstacle shapes in terms MEAN \pm HVR

\begin{tabular}{lrrr}
\hline Obstacle shape & \multicolumn{1}{c}{$F_{d y n}(\mathrm{kN})$} & \multicolumn{1}{c}{$a_{2}\left(\mathrm{~m} / \mathrm{s}^{2}\right)$} & $x_{2}(\mathrm{~cm})$ \\
\hline cos & $-0.0152 \pm 0.5350$ & $0.0770 \pm 2.3770$ & $0.0745 \pm 0.1145$ \\
half-sine & $-0.0849 \pm 0.5701$ & $-0.0970 \pm 2.6410$ & $0.0935 \pm 0.1445$ \\
half-ellipse & $-0.1313 \pm 0.6205$ & $-0.2720 \pm 2.8730$ & $0.1130 \pm 0.1760$ \\
power(1) & $-0.0250 \pm 0.5033$ & $0.0135 \pm 2.2225$ & $0.0740 \pm 0.1140$ \\
power(1.5) & $-0.0768 \pm 0.5437$ & $-0.0650 \pm 2.5280$ & $0.0880 \pm 0.1360$ \\
parabola & $-0.0964 \pm 0.5811$ & $-0.1345 \pm 2.6975$ & $0.0970 \pm 0.1510$ \\
asym. power $\left(k_{O S}, k_{R O}\right), \mathrm{CC}+\mathrm{B}, a=0.613$ & $-0.1039 \pm 0.5206$ & $-0.0980 \pm 2.5090$ & $0.0885 \pm 0.1365$ \\
asym. cos, CC+B, $a=0.613$ & $-0.0372 \pm 0.5154$ & $0.0705 \pm 2.3735$ & $0.0745 \pm 0.1145$ \\
asym. power $\left(k_{O S}, k_{R O}\right), \mathrm{CC}+\mathrm{P}, a=0.417$ & $0.0464 \pm 0.5538$ & $0.0350 \pm 2.4990$ & $-0.0860 \pm 0.1340$ \\
asym. cos, CC+P, $a=0.417$ & $-0.0038 \pm 0.5469$ & $-0.0790 \pm 2.3700$ & $-0.0740 \pm 0.1150$ \\
RANGE $(\mathrm{B} / \mathrm{P})$ & $0.1835 / 0.2444$ & $0.3650 / 0.9360$ & $0.2480 / 0.1800$ \\
\hline
\end{tabular}

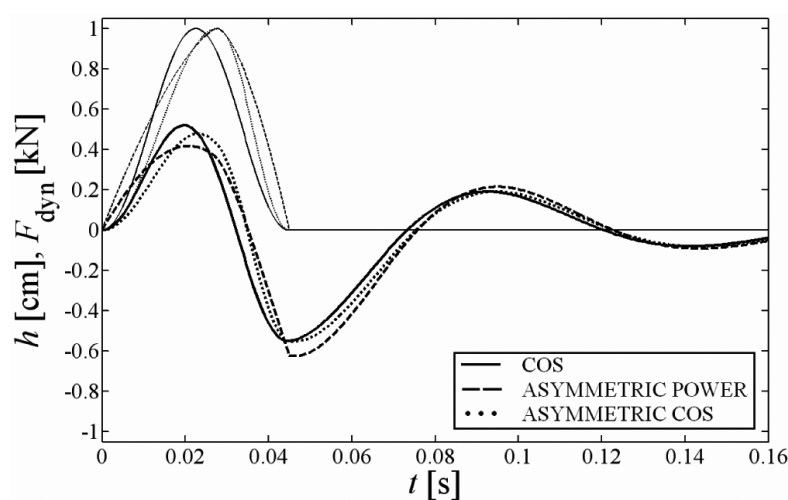

(a)

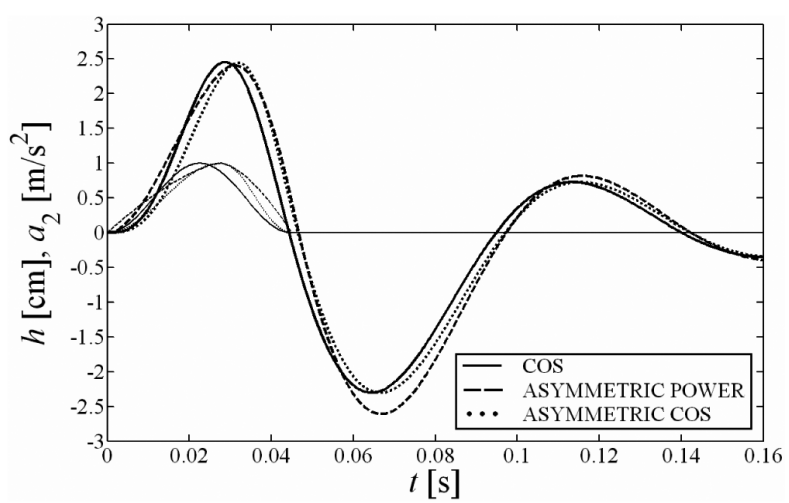

(b)

Fig. 8. Time vibration response of a quarter-car model (thick lines) to bump (thin lines): (a) dynamic tyre force, $F_{d y n}$; (b) sprung mass acceleration, $a_{2}$.

$600 \mathrm{Ns} / \mathrm{m}$, giving the corresponding undamped natural frequencies, $f_{1}=11 \mathrm{~Hz}, f_{2}=1.21 \mathrm{~Hz}$, and the damping ratio $D_{2}=0.377$.

The vehicle model response to various bump obstacle shapes in terms of vertical dynamic tyre force, $F_{d y n}$, vertical elevation, $x_{2}$, and acceleration, $a_{2}$ of the sprung mass was evaluated for $v=80 \mathrm{~km}$. It is also assumed that the tyre behaves as a point-contact follower. Dynamic loading of structures affects their durability (longevity) while for the characterization of the former (in the simplest case of stationary vibration process) the mean value, MEAN = $0.5(\mathrm{Max}+\mathrm{Min})$ and the half-range-variation, $\mathrm{HRV}=0.5(\mathrm{Max}-\mathrm{Min})$ are the decisive quantities. All obstacles considered were of the same length, $l_{O B}=1 \mathrm{~m}$, and height, $h_{O B}=1 \mathrm{~cm}$. The time response was calculated in time range from 0 to $0.45 \mathrm{~s}$, i.e. from 0 to $10 \mathrm{~m}$ for $v=80 \mathrm{~km} / \mathrm{h}$. In Table 6 , these quantities are displayed first for six selected symmetric bump models, on the next two lines, asymmetric power and cosine models for bumps with the $\operatorname{MED}(a)$ estimates are given and in the last two lines the same asymmetric pothole models are presented. Note: For symmetric obstacle shapes, the first six obstacle models are applicable for potholes when giving to the $(M E A N \pm H R V)$ values opposite signs. For the valuation of symmetric obstacle models, let us consider the cosine as the best smooth course along the whole obstacle length while the half-ellipse as the most aggressive shape on both obstacle ends, and a triangle (power(1)) with a sharp obstacle peak. As could be expected the half-ellipse shape gives greatest values both for MEAN and HVR for all response variables considered. Surprisingly, but the smallest values in the HVR variable that is decisive for the characterization of dynamic vehicle response occur for the triangle shape with distinct discontinuities in smoothness at both obstacle ends and at the maximum height/depression values. For power(1.5), which according to Table 2 fits best the empirical shapes the response variables take some higher values than for cosine but the differences are within the relevant uncertainty bands. This statement is valid for the remaining shapes as well. Notice some differences between selected response variables. While the MEAN for $F_{d y n}$ is negative for all obstacle shapes and for $x_{2}$ for all shapes is positive (for bumps, with opposite signs for potholes), for $a_{2}$ it is 
for different shapes partly positive, partly negative, obviously, in all cases the values lie within the uncertainty bands. For asymmetric shapes, additional differences follow between bumps and potholes. In Fig. 8, selected illustrative examples are shown, giving an image to the whole response courses delimited by data presented in Table 6 .

The obstacles' dimensions (height, length, etc.) distributions and statistics important for simulation of the vehicle vibration response on the obstacle were published in [8]. Further, the obstacles' dimensions empirical distributions were fitted with analytical distributions and the generator of artificial road profiles consisting of the random and the obstacle parts was designed in [10].

\section{Conclusions}

In the professional literature, a complex analysis of obstacle shapes in longitudinal road profile did not appear up to now. The choice of obstacle shapes for various interaction tasks in the system pavement-vehicle-driver is prevailingly only intuitive without accounting for real shapes. The separation of individual obstacles in the road profile records by median filtering [8] provides a suitable background for a reasonable exploitation of current empirical road profile data gathered from the LTPP program. In summary, 16590 obstacles were analysed partitioned into four sub-groups according to the asphalt-concrete vs. cement-concrete road surfaces, and positive vs. negative vertical elevations. The empirical obstacle shapes were fitted using seven symmetric and two asymmetric analytical shapes.

The main global results of processed obstacles data may be summarized as follows:

(1) Due to a very large variability of in-situ obstacle shapes, and consequently, only small differences in the applied fitting criteria, it would seem that it is in principle not important which analytical shape should be applied. Nevertheless, from the comparison of shapes alone, the power(1.5) and half-sine models can be recommended as the most suitable.

(2) Based on visualized empirical shapes the partitioning of the whole LTPP population into four groups seams to be justifiable and the differences between partial sub-groups are apparent. In spite of this statement, the best fitting functions belong - due to the great variability of empirical shapes - to a common power $(k)$ type with small differences in the values of the exponent $k$. It appears that for more precise approximations some further specification of obstacles such as the cause of their appearance and possibly further relevant circumstances would be necessary.

(3) Besides the most frequently appearing power $(k)$ shapes, also two limiting shapes were considered for comparison, viz. the half-ellipse with vertical slopes at the end points as the most aggressive shape, and cosine with zero slope at its end points as the smoothest. Both these shapes display worst closeness to the mean of empirical shapes. Nevertheless, they may be useful for the solution of special application or simulation tasks.

(4) The analysis of localization of max/min elevation positions of obstacles revealed the existence of asymmetric obstacle shapes, which were distinct, particularly with potholes on cement concrete road surface.

(5) A supplementary analysis of the vibration response of a quarter-car model with a point-follower wheel model demonstrated a high sensitivity of different response variables to the various obstacle shapes. The simulated response values in terms of variation range often differ by up to some tens of percent particularly for bumps and sprung mass accelerations.

(6) Due to moving average pre-processing of the evaluated LTPP data, a bias in the obstacle shape occurs. For the power obstacle model, this effect demonstrates as a decrease of the power exponent $k$ by 0.5 . Thus, the corresponding correction, if desired, consists in increasing the relevant exponents given in this paper by 0.5 . For the cosine shape, this bias is less pronounced and does not require a correction to be made.

\section{Acknowledgement}

This work has been partially undertaken within the grant No. 2/0075/10 of the VEGA Grant Agency of Slovak Academy of Sciences. The support of the Agency is gratefully acknowledged. Further, the authors highly appreciate the voluminous work conducted by the LTPP team and additional information provided by the LTPP Customer Service. 


\section{References}

[1] CSN 30 0560, Suspension of vehicles. Measuring of dynamic coefficients during driving of vehicles across obstacles (in Czech), The Czech Normalisation Institute, Prague, 1970.

[2] Data Pave Online, Release 20, Federal Highway Administration, Available from http://www.datapave.com, 2005.

[3] J.B. Ferris, Singular value decomposition of road events into characteristic shapes, Dynamic Systems and Control Division DSC, ASME 70 (2001), 121-126.

[4] T.S. Huang (ed.), Two-dimensional Digital Signal Processing II: Transforms and Median Filters, Springer, Berlin, 1981.

[5] S.M. Karamihas, Long-term Pavement Performance Road Profile Data, Available from http://www.umtri.umich.edu/erd/roughness/ ltpp_erd.html, 2005.

[6] O. Kropáč and P. Múčka, Effect of obstacles on the indicators of road unevenness, Proceedings of IMechE, Part D, Journal of Automobile Engineering 220 (2006), 1333-1343.

[7] O. Kropáč and P. Múčka, Effect of obstacles in the road profile on the dynamic response of a vehicle, Proceedings of IMechE, Part D, Journal of Automobile Engineering 222 (2008), 353-370.

[8] O. Kropáč and P. Múčka, Specification of obstacles in the longitudinal road profile by median filtering, ASCE Journal of Transportation Engineering, in press.

[9] O. Kropáč and P. Múčka, Properties of random component of longitudinal road profile influenced by local obstacles, International Journal of Vehicle Systems Modelling and Testing 4 (2009), 256-276.

[10] P. Múčka and O. Kropáč, Simulation of Obstacles in a Longitudinal Road Profile, Journal of Testing and Evaluation (2010), (submitted for publication).

[11] M. Mitschke, G. Klingner and H. Braun, Zulässige Amplituden und Wellenlängen herausragender Unebenheitsanteile/Einfluss von Einzelhindernissen und Periodizitäten auf Komfort, Strassen-, Fahrzeug- und Ladegutbeanspuchung sowie Fahrsicherheit (in German), Forschung Strassenbau und Strasenverkehrstechnik, Heft Nr. 710, Bundesministerium für Verkehr, Bonn, Germany, 1995.

[12] F. Öijer and S. Edlund, Identification of transient road obstacle distributions and their impact on vehicle durability and driver comfort, Vehicle System Dynamics 41 (2005), 744-753.

[13] A.V. Pesterev, L.A. Bergman, C.A. Tan and B. Yang, Assessing tire forces due to roadway unevenness by the pothole dynamic amplification factor method, Journal of Sound and Vibration 279 (2005), 817-841.

[14] M.W. Sayers, Profiles of Roughness, Transportation Research Record, No. 1260, Transportation Research Board, Washington, D.C., 1990, $106-111$. 

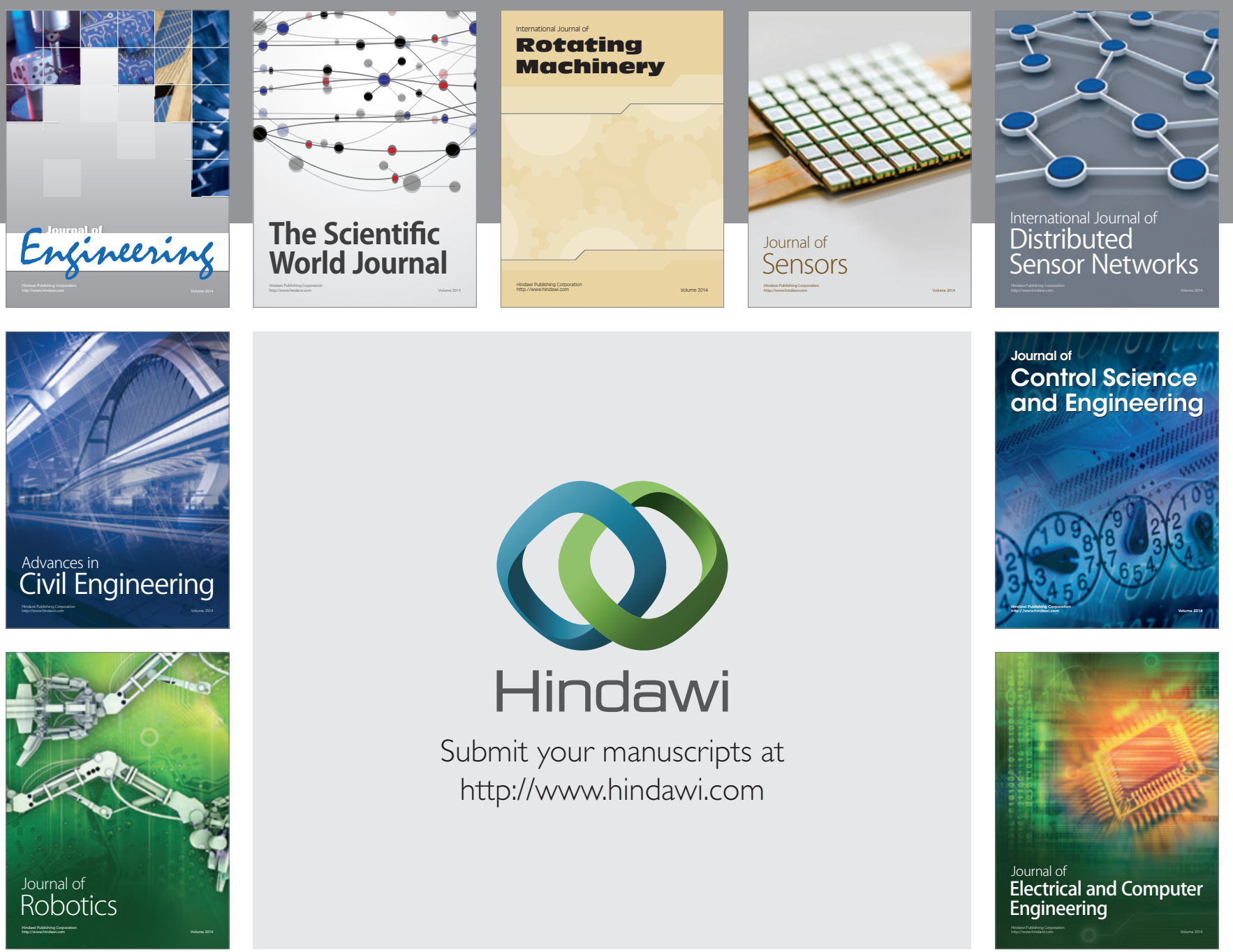

Submit your manuscripts at

http://www.hindawi.com
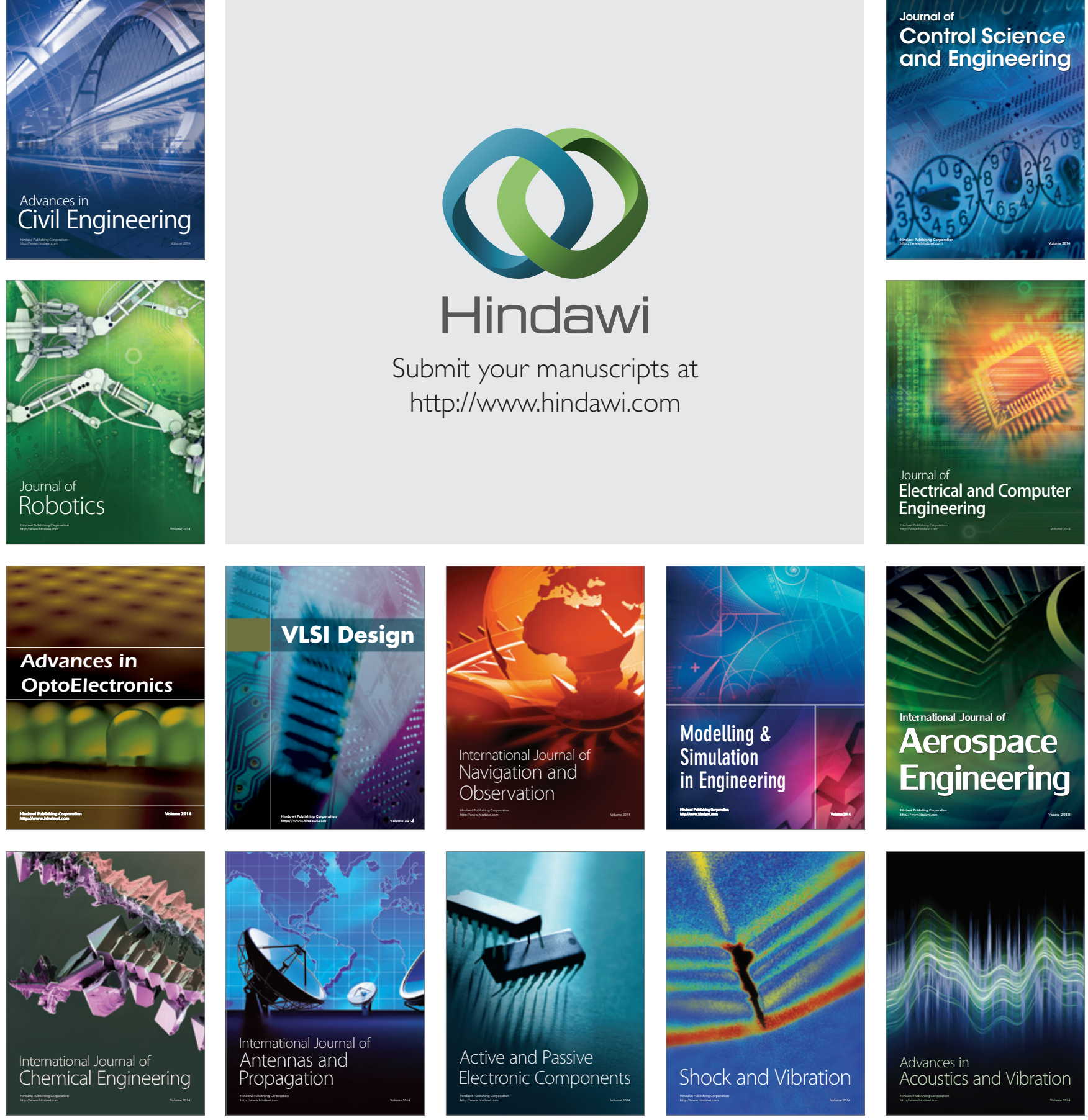